\title{
Spherically symmetric solutions of Einstein + non-polynomial gravities
}

\author{
S. Deser* \\ California Institute of Technology, Pasadena, CA 91125 and \\ Department of Physics, Brandeis University, Waltham, MA 02254, U.S.A. \\ Özgür Sarıŏlu ${ }^{\dagger}$ and Bayram Tekin ${ }^{\ddagger}$ \\ Department of Physics, Faculty of Arts and Sciences, \\ Middle East Technical University, 06531, Ankara, Turkey
}

(Dated: February 21, 2013)

\begin{abstract}
We obtain the static spherically symmetric solutions of a class of gravitational models whose additions to the General Relativity (GR) action forbid Ricci-flat, in particular, Schwarzschild geometries. These theories are selected to maintain the (first) derivative order of the Einstein equations in Schwarzschild gauge. Generically, the solutions exhibit both horizons and a singularity at the origin, except for one model that forbids spherical symmetry altogether. Extensions to arbitrary dimension with a cosmological constant, Maxwell source and Gauss-Bonnet terms are also considered.
\end{abstract}

PACS numbers: 04.20.-q, 04.20.Jb, 04.50.+h

\section{INTRODUCTION}

To better appreciate the nature of Ricci-flat geometries, particularly the fundamental exterior Schwarzschild solution of GR, it is useful to explore some alternatives, keeping as much as possible of the GR physics. The study of alternate theories is of course an enormous industry, but our interest here is to probe as simply as possible how the Schwarzschild geometry is deformed by additional terms. This will give a little more insight into the necessity for and variety of horizons, on which one could test the universality of our present black hole ideas. To be sure, we will only look under "lampposts" - models for which spherically symmetric solutions can be found explicitly. Our deviations from GR consist of those terms, non-polynomial in the Weyl tensor, whose virtue is to preserve the (first) derivative order of the GR equations in Schwarzschild gauge and to provide the simplest non-Ricci flat extensions of GR.

Our solution technique (introduced by Weyl [1] for pure GR but justified later [2, 3]) is to insert in the action a gauge fixed metric already endowed with the desired symmetries. Gauge fixing means only that the Bianchi identities become implicit, while ansatzing a spherically symmetric $g_{\mu \nu}$ will enormously simplify the labor of obtaining the field equations for the two functions on which it depends. We are assured by [2] that all solutions are

\footnotetext{
*Electronic address: deser@brandeis.edu

†Electronic address: sarioglu@metu.edu.tr

$\ddagger$ Electronic address: btekin@metu.edu.tr
} 
obtained thereby. We will also include a cosmological constant, a Maxwell source and - in $D>4$ - Gauss-Bonnet terms.

\section{THE MODELS}

In this section, we keep to $D=4$ for ease of notation. The most general spherically symmetric metric in Schwarzschild coordinates is usefully written as

$$
d s^{2}=-a(r, t) b^{2}(r, t) d t^{2}+\frac{d r^{2}}{a(r, t)}+r^{2} d \Omega_{2} .
$$

All nonvanishing components of the mixed Weyl tensor, $C_{\mu \nu}{ }^{\alpha \beta}$, are proportional to the single function $X$

$$
X(r, t) \equiv \frac{1}{r^{2}}\left(2(a-1)-2 r a^{\prime}+r^{2} a^{\prime \prime}\right)+\frac{1}{r b}\left(3 r a^{\prime} b^{\prime}-2 a\left(b^{\prime}-r b^{\prime \prime}\right)\right)+\frac{1}{b} \partial_{t}\left(\frac{1}{a^{2} b} \partial_{t} a\right) ;
$$

here primes denote radial derivatives. This means that any scalar of order $n$ in the Weyl tensor $C$ is proportional to $X^{n}$. Indeed, this fact is part of a general classification [4] of all algebraic curvature invariants of spherical geometries. This classification also informs us that the local actions that maintain the derivative order of the GR equations for the metric (1) are non-polynomial terms of the form $\left(\operatorname{tr} C^{n}\right)^{1 / n},\left(\operatorname{det} C^{n}\right)^{1 / n}$, etc. Its Ricci scalar is

$$
R=-\frac{1}{r^{2}}\left(2(a-1)+4 r a^{\prime}+r^{2} a^{\prime \prime}\right)-\frac{1}{r b}\left(3 r a^{\prime} b^{\prime}+2 a\left(2 b^{\prime}+r b^{\prime \prime}\right)\right)+\frac{1}{b} \partial_{t}\left(\frac{1}{a^{2} b} \partial_{t} a\right) .
$$

The actions we consider then are, in units of $\kappa=1$,

$$
I=\frac{1}{2} \int d^{4} x \sqrt{-g}\left(R+\beta_{n}\left|\operatorname{tr} C^{n}\right|^{1 / n}\right),
$$

where

$$
\operatorname{tr} C^{n} \equiv C_{a b}{ }^{c d} C_{c d}{ }^{e f} \ldots C_{. .}{ }^{p q} C_{p q}{ }^{a b}=\left(-\frac{1}{3}\right)^{n}\left[2+(-2)^{2-n}\right] X^{n},
$$

for $n$ copies of the Weyl tensor $C$, and there can be any number of such Weyl additions. Without loss of generality, we consider $n=2$. Defining $\sigma=\beta_{2} / \sqrt{3}$, the action (4), up to boundary terms, reduces to the almost trivial form

$$
I \rightarrow \int_{0}^{\infty} d r\left[(1-\sigma)\left(a r b^{\prime}+b\right)+3 \sigma a b\right] .
$$

Note that all the time-derivative terms have dropped out of the action. [While this does not in itself guarantee Birkhoff's theorem, the methods of [5] should do so.] The resulting two field equations decouple and give the immediate solution

$$
a(r)=\frac{1-\sigma}{1-4 \sigma}+a_{1} r^{(4 \sigma-1) /(1-\sigma)}, \quad b(r)=b_{1} r^{3 \sigma /(\sigma-1)},
$$

$\left(a_{1}, b_{1}\right)$ are integration constants and $b_{1}$ is removable by time rescaling. Note immediately that the range $1 / 4<\sigma<1$ is excluded to retain the signature. There are two special values 
of $\sigma$ : At $\sigma=1$, there is no solution at all, as is obvious from (6). To our knowledge, this is the only gravitational model without a spherical metric! For $\sigma=1 / 4$, one finds

$$
a(r)=\ln \left(\frac{r}{r_{0}}\right), \quad b(r)=\frac{1}{r},
$$

instead of (7); there is a horizon at $r=r_{0}$. The singularity at $r=0$ can be seen from

$$
R_{\mu \nu \alpha \beta} R^{\mu \nu \alpha \beta}=\frac{4}{r^{4}}\left(6-12 \ln \left(\frac{r}{r_{0}}\right)+7 \ln ^{2}\left(\frac{r}{r_{0}}\right)\right), \quad R=\frac{2}{r^{2}}\left(1-\ln \left(\frac{r}{r_{0}}\right)\right) .
$$

Both invariants vanish at infinity, and $R_{\mu \nu \alpha \beta} R^{\mu \nu \alpha \beta}$ is positive at all finite $r$. On the other hand, the curvature scalar changes its sign on the horizon. As $r \rightarrow \infty$, both $g_{00}$ and $g_{r r}$ go to 0 , so this model is unphysical.

The independent curvature invariants for the generic solution, $\sigma<1 / 4$ and $1<\sigma$, are

$$
\begin{gathered}
R_{\mu \nu \alpha \beta} R^{\mu \nu \alpha \beta}=\frac{12 r^{-4+2 /(\sigma-1)}}{(1-\sigma)^{2}(1-4 \sigma)^{2}}\left(3 \sigma^{2} r^{2 /(1-\sigma)}\left(4-2 \sigma+7 \sigma^{2}\right)\right. \\
\left.+6 a_{1} \sigma r^{(1+4 \sigma) /(1-\sigma)}\left(1-3 \sigma-3 \sigma^{2}-4 \sigma^{3}\right)+a_{1}^{2} r^{8 \sigma /(1-\sigma)}(1-4 \sigma)^{2}\left(1+5 \sigma^{2}\right)\right), \\
\left.R=\frac{6 \sigma}{r^{2}(1-\sigma)(1-4 \sigma)}\left(-3 \sigma+(4 \sigma-1) a_{1} r^{(1-4 \sigma) /(\sigma-1)}\right)\right) .
\end{gathered}
$$

For $\sigma=0$, one recovers the GR results $\left(R_{\mu \nu \alpha \beta} R^{\mu \nu \alpha \beta}=12 a_{1}^{2} / r^{6}, R=0\right)$ as $(7)$ limits to the Schwarzschild metric for $a_{1}<0$. The spatial behavior of these invariants can be mapped for the allowed ranges of $\sigma$ and compared to GR. Both scalars vanish at infinity, and diverge only at the origin for all viable $\sigma$. For physical, negative $a_{1}, R_{\mu \nu \alpha \beta} R^{\mu \nu \alpha \beta}$ is positive at all finite $r$. The curvature scalar $R$ does vanish, and changes sign, at some finite $r$, when $0<\sigma<1 / 4$ [unlike the $\sigma=1 / 4$ case, not at the horizon!]. For $\sigma<0$ and $1<\sigma, R<0$ has no sign flip.

The pure Weyl, $\sigma \rightarrow \infty$ end, exhibits slower fall-off than Schwarzschild, namely $R_{\mu \nu \alpha \beta} R^{\mu \nu \alpha \beta}=63 /\left(4 r^{4}\right), R=-9 /\left(2 r^{2}\right)$ for $a_{1}=0$. Moreover, in the pure Weyl case, one can show that the spacetime is conformally flat, $C_{\mu \nu \alpha}{ }^{\beta}=0$. That is, $X$ vanishes on Weyl shell, just as $R$ did on GR shell at $\sigma=0$. Note that our "Weyl" actions are not conformally invariant (unlike the $C^{2}$ action), not being of degree zero in the metric. Correspondingly, our conformal factor is fixed rather than arbitrary [7].

Since the asymptotic behaviors of $g_{00}$ and $g_{r r}$ differ, the equivalence principle is violated. (This is a bit loose since we have defined neither inertial nor gravitational mass. To see the difficulty of defining a "mass" in this theory, one can consider the small $\sigma$ limit, say to order $\sigma^{2}$. At this order $g_{00}$ and $g_{r r}$ have $\ln r$ terms in addition to GR's $1 / r$ parts.) However, it must be admitted that there is no qualitative surprise here, such as loss of origin singularity or horizons, apart from the $\sigma=1$ loss of solution.

\section{GENERALIZATIONS AND OTHER $D$}

In this section, we extend the above exercise to include a cosmological constant, Maxwell matter and allow general dimension:

$$
I=\frac{1}{2} \int d^{D} x \sqrt{-g}\left(R+\Lambda+\beta_{n}\left|\operatorname{tr} C^{n}\right|^{1 / n}-\frac{1}{4} F_{\mu \nu} F^{\mu \nu}\right) .
$$


The form of the metric is unchanged,

$$
d s^{2}=-a(r) b^{2}(r) d t^{2}+\frac{d r^{2}}{a(r)}+r^{2} d \Omega_{D-2} .
$$

Its curvature scalar reads

$$
R=-\frac{1}{r^{2}}\left((D-2)(D-3)(a-1)+2(D-2) r a^{\prime}+r^{2} a^{\prime \prime}\right)-\frac{1}{r b}\left(3 r a^{\prime} b^{\prime}+2 a\left((D-2) b^{\prime}+r b^{\prime \prime}\right)\right) .
$$

In $D$-dimensions, the expressions analogous to those in $D=4$ become [4]

$$
\operatorname{tr} C^{n}=\left(-\frac{D-3}{D-1}\right)^{n}\left[1-2(2-D)^{1-n}+\left(\frac{2}{(D-2)(D-3)}\right)^{n-1}\right] X^{n},
$$

where now

$$
\sigma \equiv \beta \frac{D-3}{D-1}\left|1-2(2-D)^{1-n}+\left(\frac{2}{(D-2)(D-3)}\right)^{n-1}\right|^{1 / n} .
$$

Including the Maxwell term, with $A_{\mu}=\left(A_{0}(r), 0, \ldots, 0\right)$, reduces the total action (11) to

$$
\begin{aligned}
& \frac{1}{2} \int_{0}^{\infty} d r\left[(D-2)(1-\sigma) a b^{\prime} r^{D-3}+\sigma(D-2)(D-1) a b r^{D-4}\right. \\
& \left.\quad+[(D-2)(D-3)-2 \sigma] b r^{D-4}+\Lambda b r^{D-2}+\frac{r^{D-2}}{2 b}\left(A_{0}^{\prime}\right)^{2}\right] .
\end{aligned}
$$

For generic values of the parameters in the action, the solution of the field equations arising from (16) reads

$$
\begin{aligned}
a(r)= & \frac{(D-3)(D-2)-2 \sigma}{(D-2)((D-3)-2 \sigma(D-2))}+a_{1} r^{(2 \sigma(D-2)-(D-3)) /(1-\sigma)} \\
& +\frac{q^{2} r^{6-2 D}}{2(D-2)(2 \sigma+(D-3))}+\frac{\Lambda r^{2}}{(1-2 \sigma)(D-2)(D-1)}, \\
b(r)= & b_{1} r^{\sigma(1-D) /(1-\sigma)}, \quad A_{0}^{\prime}=q b(r) r^{2-D},
\end{aligned}
$$

where $a_{1}, b_{1}$ and $q$ are integration constants.

Just as in the $D=4$ case, there are some special values for $\sigma$. The $\sigma=1$ case still excludes a solution. For $\Lambda \neq 0$, there is one additional point: For $\sigma=1 / 2$,

$$
a(r)=-\frac{D^{2}-5 D+5}{D-2}+\frac{2 \Lambda}{(D-2)} r^{2} \ln a_{1} r+\frac{q^{2}}{2(D-2)^{2}} r^{6-2 D}, \quad b(r)=b_{1} r^{1-D} .
$$

For $q \neq 0, \sigma=-(D-3) / 2$ is a special point for which

$$
a(r)=\frac{1}{D-2}+\frac{\Lambda r^{2}}{(D-1)(D-2)^{2}}-\frac{q^{2}}{(D-2)(D-1)} r^{6-2 D} \ln a_{1} r, \quad b(r)=b_{1} r^{D-3} .
$$

The remaining special cases are $\sigma=(D-3) /(2(D-2))$ for which

$$
a(r)=\frac{\Lambda r^{2}}{(D-1)}+\frac{q^{2}}{2(D-3)(D-1)} r^{6-2 D}+\frac{2(D-3)^{2}}{D-2} \ln a_{1} r, \quad b(r)=b_{1} r^{3-D},
$$

and finally $\Lambda=q=0$ reduce (17), (18) to the pure "Weyl case"

$$
a(r)=\frac{1}{(D-2)^{2}}+a_{1} r^{4-2 D}, \quad b(r)=b_{1} r^{D-1} .
$$

A fair conclusion of this Section's various generalizations to include Maxwell matter, a cosmological term and arbitrary dimension is that they have not led to any substantial new surprises beyond the original model's. 


\section{SUMMARY:}

We have investigated the class of gravitational additions to GR that preserves GR's derivative order in the spherically symmetric problem: it is uniquely found to be $\left(\operatorname{tr} C^{n}\right)^{1 / n}$. This choice was made - apart from its easy solubility - to see how radically a physically natural extension of GR alters the Schwarzschild metric's properties. Our findings for the simplest $D=4$ story led to rather strange metrics, which however retain the former's qualitative properties: horizon and origin singularity, apart from the complete loss of a solution at $\sigma=1$. These properties are essentially maintained by our further generalizations.

\section{Acknowledgments}

S.D. was supported in part by NSF grant PHY04-01667, and thanks Dr. G. Conrad for a stimulating contribution. The work of Ö.S. and B.T. is partially supported by the Scientific and Technological Research Council of Turkey (TÜBITTAK). B.T. is also partially supported by the "Young Investigator Fellowship" of the Turkish Academy of Sciences (TÜBA) and by the TÜBİTAK Kariyer Grant 104T177.

\section{APPENDIX: GAUSS-BONNET AUGMENTED MODEL}

Here we add a Gauss-Bonnet term to (11) which, of course, only contributes for $D \geq 5$ :

$$
\begin{aligned}
\frac{\gamma \sqrt{-g}}{\Omega_{D-2}}\left(R^{2}-4 R_{\mu \nu}^{2}+R_{\mu \nu \alpha \beta}^{2}\right)= & \gamma\left[( D - 2 ) ( D - 3 ) r ^ { D - 4 } \left(2 a a^{\prime} b+4 a^{2} b^{\prime}+(D-4) a^{2} b / r\right.\right. \\
& \left.\left.-2(D-4) a b / r-2 a^{\prime} b-4 a b^{\prime}+(D-4) b / r\right)\right]^{\prime} \\
& -\gamma(D-4)(D-3)(D-2) r^{D-5}(a-1)^{2} b^{\prime}
\end{aligned}
$$

The bracketed term is a total divergence, so the overall reduced action is still of first derivative order

$$
\begin{array}{r}
\frac{1}{2} \int_{0}^{\infty} d r\left[(D-2)(1-\sigma) a b^{\prime} r^{D-3}+\sigma(D-2)(D-1) a b r^{D-4}\right. \\
\left.+[(D-2)(D-3)-2 \sigma] b r^{D-4}+\Lambda b r^{D-2}+\frac{r^{D-2}}{2 b}\left(A_{0}^{\prime}\right)^{2}-\lambda r^{D-5}(a-1)^{2} b^{\prime}\right],
\end{array}
$$

here $\lambda \equiv \gamma(D-4)(D-3)(D-2)$. Without the "Weyl" term, the general solution is well known [6], [3]. As a specific example, consider $D=5$ with $\Lambda=0$ and $A_{0}=0$. For the generic values of the parameter $\sigma$ (including the $\sigma=1$ case, which is here resuscitated!), one can obtain a solution: The function $b(r)$ can be solved in terms of $a(r)$; however the solution for $a(r)$ itself is best not displayed. There is one special case $\sigma=1 / 3$ for which the solution is "somewhat" presentable:

$$
a(r)=1+\frac{1}{\lambda} r^{2}-\frac{4}{3}\left(1+a_{1}+W\left(-e^{-1+3 r^{2} /(4 \lambda)}\right)\right), \quad b(r)=e^{\left(b_{1}-3 a(r)\right) / 4},
$$


where $W(z)$ denotes the Lambert $W$ function, i.e. $z=W(z) e^{W(z)}$ and $W(z)$ is single valued for real $z \geq-1 / e$ with $W(z) \geq-1$ demanded.

[1] H. Weyl, "Space-Time-Matter", New York: Dover, (1951).

[2] R.S. Palais, Comm. Math. Phys. 69, 19 (1979).

[3] S. Deser and B. Tekin, Class. Quant. Grav. 20, 4877 (2003) [arXiv:gr-qc/0306114].

[4] S. Deser and A.V. Ryzhov, Class. Quant. Grav. 22, 3315 (2005) [arXiv:gr-qc/0505039].

[5] S. Deser and J. Franklin, Am. J. Phys. 73, 261 (2005) [arXiv:gr-qc/0408067].

[6] D.G. Boulware and S. Deser, Phys. Rev. Lett. 55, 2656 (1985).

[7] There is a slight formal caveat here, which we have not pursued in detail, given the assurance provided by [2]. Stated succinctly, if one varies the full action $\int d^{4} x \sqrt{-g} \sqrt{\operatorname{tr} C^{2}}$, the resulting field equation contains a term of the form $\nabla \nabla\left(C / \sqrt{\operatorname{tr} C^{2}}\right)$, which seems to have the form $0 / 0$ in $C=0$ spaces (the other terms vanish there). However, we believe that performing the differentiations and then going to our ansatz will result in the equations we have found above. In any case, the Einstein term acts as a regulator while the $\sigma \rightarrow \infty$ limit is taken. 